\title{
FEM $\times$ DEM multiscale modeling: Model performance enhancement from Newton strategy to element loop parallelization
}
A. Argilaga
J. Desrues
S. Dal Pont
G. Combe
D. Caillerie

Laboratoire 3SR, Université Grenoble Alpes, UMR CNRS 5521, 38000 Grenoble, France

Correspondence

S. Dal Pont, Laboratoire 3SR, Université Grenoble Alpes, UMR CNRS 5521, 38000 Grenoble, France.

Email: stefano.dalpont@3sr-grenoble.fr

\begin{abstract}
Summary
This paper presents a multiscale model based on a FEM $\times$ DEM approach, a method that couples discrete elements at the microscale and finite elements at the macroscale. $F E M \times D E M$ has proven to be an effective way to treat real-scale engineering problems by embedding constitutive laws numerically obtained using discrete elements into a standard finite element framework. This proposed paper focuses on some numerical open issues of the method. Given the nonlinearity of the problem, Newton's method is required. The standard full Newton method is modified by adopting operators different from the consistent tangent matrix and by developing ad-hoc solution strategies. The efficiency of several existing operators is compared, and a new and original strategy is proposed, which is shown to be numerically more efficient than the existing propositions. Furthermore, a shared memory parallelization framework using OpenMP directives is introduced. The combination of these enhancements allows to overcome the $\mathrm{FEM} \times \mathrm{DEM}$ computational limitations, thus making the approach competitive with classical FEM in terms of stability and computational cost.
\end{abstract}

\section{KEYWORDS}

FEM $\times$ DEM, multiscale, Newton, numerical homogenization, parallelization

\section{1 | INTRODUCTION}

Multiscale numerical models allow to have an insight into the microscale origins of complex phenomena such as localization and anisotropy in mechanical problems. ${ }^{1-5}$ This allows to avoid the use of phenomenological constitutive laws by obtaining the material properties directly from a microscale simulation. The idea of FEM $\times$ DEM is to solve a continuum boundary value problem (BVP) at the macroscale while obtaining the constitutive material behavior from a DEM microscale in a fully coupled hierarchical multiscale method. Some early works, ${ }^{6-8}$ have put in evidence the potential of the method to provide a refined description of complex constitutive behaviors into real-scale computations. Indeed, FEM $\times$ DEM methods allow to couple the advantages of discrete elements (ie, a numerical constitutive law for a complex material behavior can be obtained) and the efficiency of finite elements. Later works have enhanced and extended this approach to the study of anisotropy, ${ }^{9,10}$ granular cohesion, ${ }^{11}$ material heterogeneity,${ }^{12}$ real-scale engineering applications, ${ }^{13,14}$ more realistic constitutive behaviors using 3-dimensional DEM, ${ }^{15,16}$ and macroscale hydro-mechanical coupling. ${ }^{16,17}$ More recently, Liu et $\mathrm{a}^{15}$ have embedded nonlocal regularization at the macroscale, and Guo and $\mathrm{Zhao}^{18}$ have developed a full micro-macro 3-dimensional approach. However, despite these latest developments, some open 
issues in the development of the method can be identified. The time dependency and nonlinearity of the problem requires an iterative Newton's scheme and the definition of the tangent operator. Full Newton's method is associated to the consistent tangent operator (CTO) that, in the case of a complex constitutive behavior, is usually obtained by derivation, ie, making use of a perturbation method. However, the use of discrete elements at the microscale intrinsically leads to numerical instabilities, ie, the noisy constitutive law by numerical homogenization of a 2-dimensional (2D) DEM model gives, as a result, a function with noncontinuous derivative after the peak. The first objective of this paper is to mitigate this issue by proposing and discussing alternative new operators and introducing quasi-Newton strategies that prove to be robust, stable, and numerically efficient. Among these, an original solution developed in this present work, namely, the DEM-based quasi-static operator (DEMQO) presented hereafter, represents the most efficient approach in terms of computational cost. Furthermore, parallelization paradigms based on a shared memory framework using OpenMP directives are introduced: this overcomes the CPU time issue due to the integration of the microscale. The combination of these enhancements allows to relieve the FEM $\times$ DEM computational limitations, thus making the approach suitable for engineering problems in terms of stability and computational cost.

This paper is organized as follows. In Section 2, the FEM and DEM theories are presented as well as the coupling FEM $\times$ DEM. In Sections 3, 4, and 5, the alternative operators are presented with performance results shown in Section 6 . Section 7 is devoted to the parallelization. This paper ends with conclusions in Section 8. In Appendices A, B, and C, the needed developments to be obtained by the operators are presented.

\section{2 | FEM $\times$ DEM MODEL}

We consider the quasi-static evolution of a continuous medium in a large deformation; the anelastic constitutive equation that is issued from a DEM computing shares features, at least from a numerical point of view, with other constitutive equations of geomechanics, eg, elastoplasticity and hypoplasticity. The numerical simulation, performed in the framework of finite elements, is based on a time-stepping method. At a given time step, the balance equation, fulfilled at the end of the step, reads in virtual power formulation as follows:

$$
\forall \vec{w}^{*},-\int_{\Omega^{f}} \boldsymbol{\sigma}^{f}: \epsilon\left(\vec{w}^{*}\right) \mathrm{d} v+\int_{\partial \Omega^{f}} \vec{B}^{f} \cdot \vec{w}^{*} \mathrm{~d} s=0 .
$$

A quantity with a superscript $i$ or $f$ refers to the value of that quantity at the beginning and at the end of the step, respectively. $\Omega^{f}$ is the unknown configuration of the medium at the end of the step, $\vec{B}^{f}$ is the force density applied on the boundary $\partial \Omega^{f}$, and $\epsilon\left(\vec{w}^{*}\right)$ denotes the symmetric part of the gradient of the virtual velocity field $\vec{w}^{*}$. One should notice that the bulk force density is neglected here. According to the problem in view, $\vec{B}^{f}$ can be partly known or partly unknown.

The constitutive equation provides the Cauchy stress $\boldsymbol{\sigma}^{f}$ as a function of the deformation tensor $\boldsymbol{F}^{f}$ that reads

$$
\boldsymbol{F}^{f} \mapsto \boldsymbol{\sigma}^{f}=S\left(\boldsymbol{F}^{f}\right) .
$$

The BVP, ie, nonlinear in general, is solved using the iterative Newton's method. At each iteration, the equations are expanded up to the first order with respect to the unknowns, which are mainly the displacement field from $\Omega^{i}$ to $\Omega^{f}$, the stress $\boldsymbol{\sigma}^{f}$, and possibly some parts of $\vec{B}^{f}$. Then, the corresponding linear problem is solved, and the unknowns are updated, ready for the following iteration. The strict application of the Newton's method needs to differentiate $S$ with respect to $\boldsymbol{F}^{f}$; the gradient of $S$, often called the "consistent operator", is denoted as $C^{C T O}$ as follows:

$$
\mathrm{d} \boldsymbol{\sigma}=S\left(\boldsymbol{F}^{f}+\mathrm{d} \boldsymbol{F}^{f}\right)-\mathcal{S}\left(\boldsymbol{F}^{f}\right)=C^{C T O}: \mathrm{d} \boldsymbol{F}^{f}+\cdots .
$$

In this paper, different alternative operators are considered in place of the consistent one, and for those operators, the differential relation of Equation (3) is written generically as follows:

$$
\mathrm{d} \boldsymbol{\sigma}=C: \mathrm{d} \boldsymbol{F} .
$$

Apart from some simple cases for which a closed-form expression of tensor $C$ can be found, the determination of $C$ is usually performed numerically using a perturbation method. In the case of a $2 \mathrm{D}$ problem, the dimension of the space of $\boldsymbol{F}^{f}$ is $4 ; \boldsymbol{F}^{f}$ is therefore perturbed in 4 independent directions, for instance, the directions given by the tensors $\boldsymbol{\Lambda}^{n m}, n$, and $m=1,2$ are defined as

$$
\Lambda_{k l}^{m n}=\delta_{m k} \delta_{n l},
$$

where $\delta$ is the Kronecker symbol. 
Then, the components

$$
C_{i j m n}=\frac{S_{i j}\left(\boldsymbol{F}^{f}+\epsilon \boldsymbol{\Lambda}^{m n}\right)-S_{i j}\left(\boldsymbol{F}^{f}\right)}{\epsilon}
$$

are computed. Assume $S$ to be differentiable that determines $C$. According to the way it is performed, the computation of $C$ can be more or less time consuming.

In this presently studied case, the determination of $\boldsymbol{\sigma}^{f}$ in terms of $\boldsymbol{F}^{f}$ is particular in the sense that it is not provided by the integration of an incremental (or partly incremental) constitutive equation on a time step (as in the case of hypoplasticity or elastoplasticity) but by the displacement field of a spatially periodic assembly of interacting grains, ie, the DEM computation. The periodicity condition means that, at each step of its evolution, the geometry assembly is periodic. The positions of the grains are given by those of the grains of a subset, called base cell, translated by vectors $v_{1} \vec{Y}^{1}+v_{2} \vec{Y}^{2}$ (in 2D), with $v_{1}$ and $v_{2}$ being the relative integers; $\vec{Y}^{1}$ and $\vec{Y}^{2}$, ie, assumed to be linearly independent, are the periodicity vectors (see Figure 1). To determine the motion of the grains of the whole assembly, it is sufficient to determine that of the grains of the base cell taking into account the possible interactions of those grains with those of the adjacent cells, ie, the cells for which $v_{1}$ and $v_{2}$ are in $\{-1,0,1\}$. The rotations of grains are supposedly periodic. The motion of the grains of the base cell is determined by numerically integrating Newton's dynamic equations, the grains interacting by contact forces that are partly elastic and that satisfy the Signorini-Coulomb conditions of friction. Some cohesion can be taken into account, yet interaction torques are disregarded. The kinematic data of the motion of grains are the time $\tau$ functions $\vec{Y}^{1}(\tau)$ and $\vec{Y}^{1}(\tau)$, the unknowns are the displacements and rotations of the grains as well as the interaction forces.

For the DEM computing of relation (2), the $\tau$ functions $\vec{Y}^{1}(\tau)$ and $\vec{Y}^{1}(\tau)$ are taken in the form $\vec{Y}^{i}(\tau)=F(\tau) \cdot \vec{Y}_{R}^{i}, i=1,2$, where $\vec{Y}_{R}^{1}$ and $\vec{Y}_{R}^{2}$ are the initial (with respect to the macroscopic evolution) periodicity vectors and where the second-order tensor function $\boldsymbol{F}(\tau)$ is given as

$$
\boldsymbol{F}(\tau)= \begin{cases}\boldsymbol{F}^{i}+\frac{\tau}{\tau_{1}}\left(\boldsymbol{F}^{f}-\boldsymbol{F}^{i}\right) & 0 \leq \tau \leq \tau_{1} \\ \boldsymbol{F}^{f} & \tau_{1}<\tau\end{cases}
$$

where $\tau_{1}$ is the duration of the progressive loading of the cell by the macroscopic deformation gradient. At the end of that period, the grains of the base cell are not necessarily balanced; therefore, the computing is carried on until the grains of the base cell are balanced (ie, the ratio between the maximum unbalanced force measured for grains in the assembly, relatively to the typical normal force, is smaller to $10^{-3}$ ) in such a way that the DEM computation should be consistent with the quasi-static feature of the macroscopic modeling. To speed up this relaxation phase, a damping term can be added to the interacting forces.

At the end of the computing, the DEM provides the positions $\vec{r}^{n}$ of the grains of the base cell numbered by $n$ and the contact forces $\vec{f}^{m / n}$ and $\vec{f}^{m / n}$ being the force applied on the grain $n$ by the grain $m$. The Cauchy stress tensor is determined according to the Cauchy-Poisson formula as follows:

$$
\boldsymbol{\sigma}^{f}=\frac{1}{\left|Y_{a}\right|} \sum_{(n, m) \in \mathcal{I}} \vec{f}^{m / n} \otimes\left(\vec{r}^{m}-\vec{r}^{n}\right),
$$

where $\left|Y_{a}\right|$ is the area of the 2D packing and $\mathcal{I}$ denotes the set of couples of grains being in contact. It can be seen that the DEM computation enables to determine $\boldsymbol{\sigma}^{f}$ in terms of $\boldsymbol{F}^{f}$, which defines the function $S$ of (2), which is possible due the quasi-static conditions imposed to the DEM model (see Equation (7)).

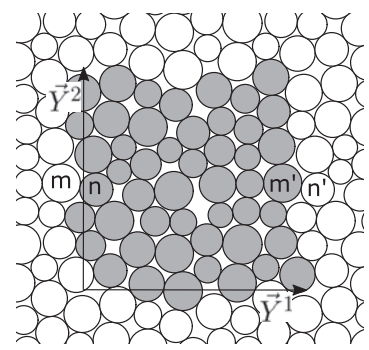

FIGURE 1 Elementary cell and periodicity vectors; $m$ and $n$ are 2 grains in contact; $m^{\prime}$ and $n^{\prime}$ are the equivalent grains in the neighbor cell 


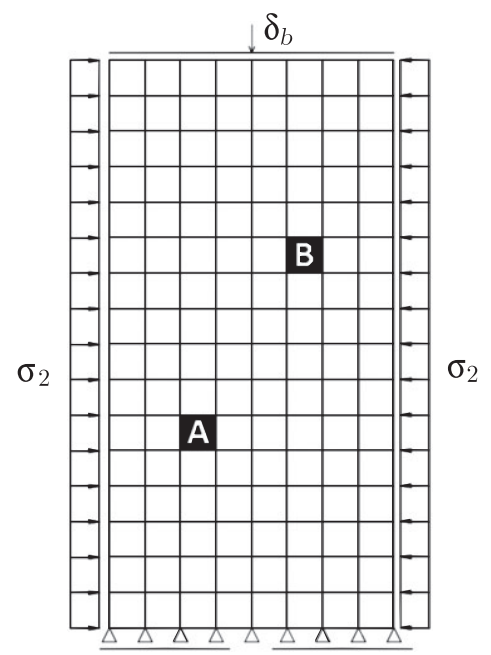

FIGURE 2 Biaxial test geometry and boundary conditions; $\sigma_{2}$ is the confining pressure; $\delta_{b}$ the imposed displacement. The concerned Gauss points are located in elements A and B

In the following sections, different Newtons' operators will be presented and compared. Section 3 will introduce the standard perturbation-based operators. Section 4 will present the standard Kruyt operator together with some possible enhancements of this operator. Section 5 will be devoted to the development of a new elastic-based numerical (ie, DEMQO) and analytic (ie, PSTLO [Prestressed truss-like operator]) operator.

All the numerical tests are performed on a biaxial test BVP. The biaxial test configuration is chosen because of its extensive use in numerical simulations and experiments (available bibliography, for example, the work Mokni and Desrues ${ }^{19}$ and Guo and $\mathrm{ZhaO}^{20}$ ). Furthermore, despite the initial homogeneity of the problem and, in fact, because of that, the biaxial test constitutes a particularly challenging BVP for numerical modeling. The emergence of a localization pattern, if any, is not prescribed by some initial heterogeneity of the material or of the stress field. Ideally, the locus of the emergence of one or several shear bands within the specimen is physically undetermined, unlike other characteristics as the orientation or the thickness of the band. In experiments, the actually emerging pattern results from subtle imperfections of the tested specimen; in silico, it will result of the noise of the numerical algorithms, but the convergence toward a given solution among a large choice of possible ones can be much more difficult than in the case of heterogeneous or initially biased BVP. ${ }^{12}$

In the forthcoming numerical simulations, a displacement controlled monotonic compression $\delta_{b}$ is applied together with a confining constant pressure, ie, equal to the initial value $\sigma_{2}$. It is worth noting that in the forthcoming analysis, gravity forces are not considered (being negligible in the context of the present study), thus making the units not relevant (it can also be observed that no length appears in Equation (2)). The height/width ratio in the proposed problem is equal to 2 . The microscale consists in 400 circular particles assembled with cohesion and friction in the contacts. The numerical study is based on four 128-element Gauss points and 8-noded-element FEM mesh (see Figure 2). The BVP is closed by restricting all the displacements in one node to avoid kinematic indetermination. Force and displacement conditions are used as convergence criteria in the Newton method, FNORM/RNORM being the force, and UNORM/DNORM being the displacement variable. Their meaning will be made explicit later in this article. PRECU is the threshold of precision for the displacement criteria.

The numerical values of the Newtons' operators are calculated for 2 elements of the 128-element simulation (see Figure 2). These 2 elements ( $A$ and B) undergo the same loading path, and given the absence of localization at the present configuration (first loading step), they should give, apart from numerical precision, identical stress-strain state to the 2 points.

\section{3 | PERTURBATION-BASED OPERATORS}

In this section the CTO, which was introduced in Equation (6), and a time average named as auxiliary elastic operator (AEO) will be presented. 


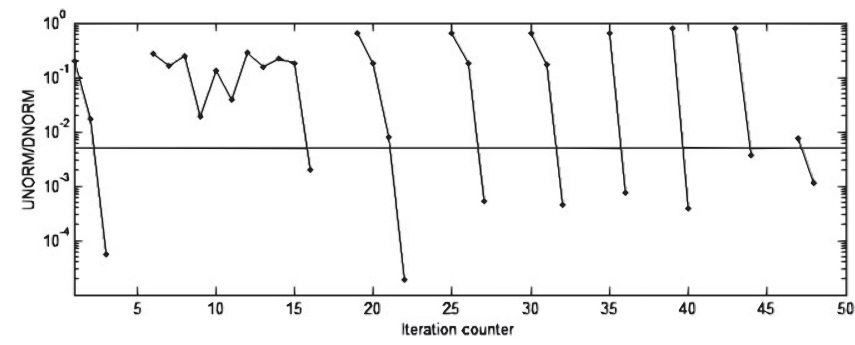

FIGURE 3 Displacement convergence profiles (UNORM/DNORM) for the first 10 time steps of a biaxial test with a maximum axial deformation less than $0.2 \%$ using consistent tangent operator. The horizontal line indicates the precision threshold for the UNORM/DNORM values; PRECU $=0.005$

\section{1 | Consistent tangent operator}

The CTO is obtained using the perturbation method in Equation (6). Four small perturbations, ${ }^{*} \in \boldsymbol{\Lambda}^{m n}, n, m=1,2$, of the form (5) are successively added to the final value $\boldsymbol{F}^{f}$ of the macroscopic deformation gradient and the corresponding stress tensors $\boldsymbol{\sigma}^{f}$ are calculated for each of them. According to Equation (7), the perturbations $€ \boldsymbol{\Lambda}^{m n}$ change the path of $\boldsymbol{F}$ along which the DEM integration is performed; that means that the calculation of the CTO needs 4 full DEM computations for each iteration of the Newton's method at each Gauss point of the FEM discretization.

When applied to a smooth enough function, the convergence of the Newton's method is proved to be quadratic; therefore, the CTO should provide that quadratic convergence. In general, the convergence obtained using it in a FEM $\times$ DEM model is not quadratic unless the state is nearly elastic. Furthermore, convergence is not always achieved.

This is partially due to the noisy behavior of the constitutive law provided by the DEM. Indeed, a noisy response degrades the precision of the numerical derivation performed to compute the CTO, when a significant number of contacts are prone to slide in the elementary volume. Conversely, when the behavior in the domain is largely elastic (within the considered loading increment), quadratic convergence may still be obtained if a limited number of grain contacts slide in some elementary volumes. This is put in evidence in Figure 3 , in which the profiles of convergence (UNORM/DNORM) ${ }^{\dagger}$ of a biaxial compression test show quadratic velocity except for the second iteration where oscillations are more evident.

The softening causes the problem to be ill-posed, thus inducing the degradation of the Newton method efficiency. ${ }^{*}$ The time $\operatorname{cost}^{\S}$ of the FEM $\times$ DEM model forces us to stop the simulation before convergence is found, ie, better numerical approaches are needed.

In Equations (9) and (10) matrix $\underline{\underline{C}}$ is given for the 2 Gauss points $\mathrm{A}$ and B, respectively. Point A (see Figure 2) gives the expected ${ }^{\text {II }}$ Newton operator coefficients (see Equation (9)), whereas Gauss point B presents very different values (see Equation (10)). Such a discrepancy is systematically observed using the CTO operator and can be due to the granular assembly noisy response and the related bifurcation behavior that might be taking place even at such an early loading stage. Other operators rather than the CTO are therefore needed to avoid this issue. Furthermore, we have

$$
\begin{aligned}
\underline{C}_{A}^{C T O} & =\left(\begin{array}{cccc}
900.3 & -16.4 & -22.1 & 73.8 \\
-14.9 & 384.4 & 418.8 & -6.0 \\
-14.9 & 384.4 & 418.8 & -6.0 \\
79.3 & -12.8 & -9.9 & 906.1
\end{array}\right) \\
\underline{\underline{C}}_{B}^{C T O} & =\left(\begin{array}{cccc}
-2077.4 & -2789.1 & 493.8 & -991.8 \\
3799.7 & -165.0 & 391.5 & -9341.1 \\
3799.7 & -165.0 & 391.5 & -9341.1 \\
-2678.0 & 3008.6 & -4965.9 & -7326.5
\end{array}\right) .
\end{aligned}
$$

\footnotetext{
*Small enough to be considered infinitesimal compared to the magnitude of the loading step.

DNORM is the norm of the nodal displacements in the current iteration, and UNORM is the norm of the nodal displacements increment in the current step.

${ }^{\ddagger}$ From a theoretical perspective, a negative stiffness coefficient is not possible because of thermodynamic considerations. ${ }^{21}$ Therefore, materials whose global behavior exhibits negative stiffness are unstable. However, if only some part of the global structure presents negative stiffness and the surrounding is stiff enough, it can absorb the instabilities and the structure can be stable. ${ }^{21}$

$\S$ In classical analytic law models, a nonconvergent Newton method can eventually converge to the solution in the next iteration; in FEM $\times$ DEM the computational time devoted to the integration of the law is dependent of the strain input, which can be very large in case of nonconvergence of the Newton method.

IValidated with the results directly obtained from the DEM code.
} 
It is worth noting that in Equations (9) and (10) (and analogous equations of the following), matrices $\underline{\underline{C}}$ represent a fourth-order tensors $C_{i j k h}$ according to

$$
\underline{\underline{C}}=\left(\begin{array}{llll}
C_{1111} & C_{1112} & C_{1121} & C_{1122} \\
C_{1211} & C_{1212} & C_{1221} & C_{1222} \\
C_{2111} & C_{2112} & C_{2121} & C_{2122} \\
C_{2211} & C_{2212} & C_{2221} & C_{2222}
\end{array}\right),
$$

which corresponds to the notation of second-order tensors as column vectors. For instance, in matrix notation, Equation (3) reads

$$
\left(\begin{array}{c}
\mathrm{d} \sigma_{11}^{f} \\
\mathrm{~d} \sigma_{12}^{f} \\
\mathrm{~d} \sigma_{21}^{f} \\
\mathrm{~d} \sigma_{22}^{f}
\end{array}\right)=\underline{\underline{C}}\left(\begin{array}{l}
\mathrm{d} F_{11}^{f} \\
\mathrm{~d} F_{12}^{f} \\
\mathrm{~d} F_{21}^{f} \\
\mathrm{~d} F_{22}^{f}
\end{array}\right) .
$$

\section{2 | Auxiliary elastic operator}

This operator was developed in the work of Nguyen, ${ }^{10}$ and it was used as an alternative to the CTO. The stress evolution in a single Gauss point suggests that the irregularities are restricted only to some iteration. This can be put down to the DEM behavior that presents instabilities only when a chain of grains slide occurs, which happens in a systematic way during a monotonic loading path. In order to provide a smoother evolution of the operator, a time average over several well-converged steps is performed at the beginning of the loading. This provides an operator (see Equation (12)), which can be adopted for the given Gauss point during the rest of the simulation.

However, as the operator remains unchanged, it does not take into account the history of the material; therefore, it is not tangent to the law once we leave the prepeak regime. Consequently, the resulting quasi-Newton method will never present quadratic convergence. Nevertheless, no perturbations are needed after obtaining the average so it only needs one-fifth of the time per Newton iteration compared to the CTO. The AEO allows for more stable simulations beyond the stress peak (see Figure 4) as follows:

$$
\underline{\underline{C}}^{A E O}=\left(\begin{array}{cccc}
880.3 & -19.0 & -18.27 & 86.9 \\
-13.3 & 379.8 & 415.11 & -15.5 \\
-13.3 & 379.8 & 415.11 & -15.5 \\
99.3 & -15.1 & -17.05 & 886.9
\end{array}\right) \text {. }
$$

\section{4 | KRUYT OPERATORS}

Another way of obtaining the Newton operator comes within a rougher method of homogenization that consists in considering that the displacements $\vec{u}^{n}$ and the rotations $\mathrm{d} \theta^{n}$ of the grains are those of the underlying continuous macroscopic medium. As stressed out in Appendix C, this method of homogenization does not make use of the balance equations of the grains, and if applied to a linear elastic continuum, it yields to a macroscopic elastic module that is the average of the constituent modules.

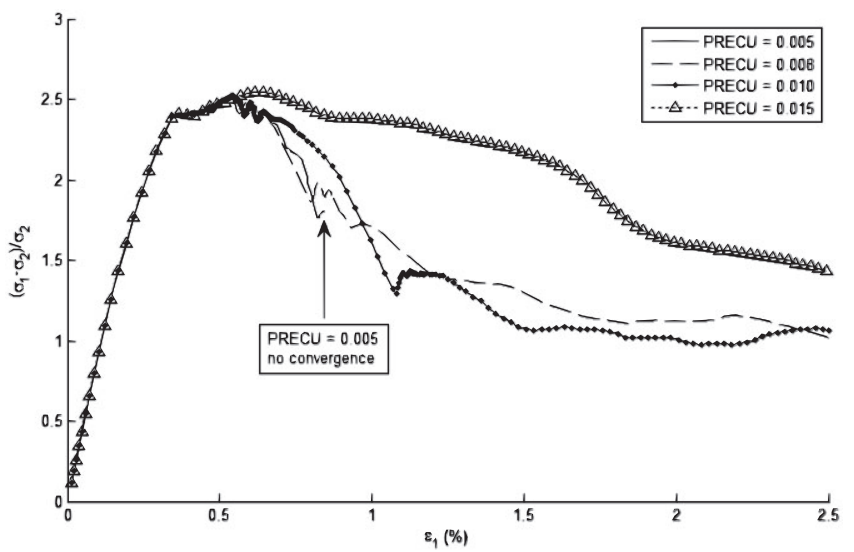

FIGURE 4 Strain stress curves for a biaxial compression FEM $\times$ DEM simulation using the auxiliary elastic operator. The tests with precision lower than PRECU $=0.005$ present good convergence 
Kruyt's operator has already been used by other authors in a FEM $\times$ DEM formulation, for example, Guo and Zhao ${ }^{13}$ proposed an upper bound Kruyt operator (UKO). Other Kruyt-like operators can be obtained by introducing some fitting coefficients derived by the actual CTO. In this work, we will introduce the upper bound corrected Kruyt operator (UCKO) and the UCKO with 2 degrees of freedom (UCKO 2DOF). Furthermore, Kruyt approach can be improved by considering additional DOF, such as the rotations of the grains and the prestresses, that yields to the auxiliary operator denoted as Kruyt augmented operator (KAO).

\section{1 | Upper bound Kruyt operator}

The elastic operator proposed by Kruyt and Rothenburg ${ }^{22}$ was introduced here. Kruyt proposed 2 extremum principles to obtain the upper and lower bounds of the elastic moduli of a granular assembly. The extremum principle used in this work is the one corresponding to the upper bound of the elastic moduli. This operator is already used in a multiscale FEM $\times$ DEM approached by Guo and Zhao. ${ }^{13}$ The UKO is obtained assuming that the displacements of the grains are those of an homogeneously strained medium, subjected to the strain $\boldsymbol{E}$. Consequently, the difference $\Delta \vec{u}^{c}$ of the grain displacements of a contact linearly depends on $\boldsymbol{E}$. That, together with the assumption that the contact force is elastic and proportional to the difference $\Delta \vec{u}^{c}$, yield that the stress reads $\boldsymbol{\sigma}=C^{U K O}: \boldsymbol{E}$. According to the Cauchy-Poisson formula for the stress, $C^{U K O}$ appears as a summation over all the contacts taking in consideration the orientation and stiffness (as shown in Equation (13)). Cohesion forces, preexisting forces, and rotations of the grains are not taken into account.

From the work of Guo and Zhao, ${ }^{13}$ the tensor $C^{U K O}$ in Equation (4) is in the notations of the present text as follows:

$$
C^{U K O}=\frac{1}{\left|Y_{a}\right|} \sum_{c \in \mathcal{I}}\left(l^{c}\right)^{2}\left(k_{n}\left(\vec{e}^{c} \otimes \vec{e}^{c}\right) \otimes\left(\vec{e}^{c} \otimes \vec{e}^{c}\right)+k_{t}\left(\vec{t}^{c} \otimes \vec{e}^{c}\right) \otimes\left(\vec{t}^{c} \otimes \vec{e}^{c}\right)\right),
$$

where $\vec{e}^{c}$ and $l^{c}$ are the unit vector and the length of the branch vector connecting the centers of 2 grains in contact, $\vec{e}^{c}$ is the normal vector to the contact and the tangent $\vec{t}^{c}$, and $k_{n}$ and $k_{t}$ are the normal and tangential contact stiffnesses (more details are given in Appendix C).

Given its elastic definition, the operator provides stability. However, not being a CTO, quadratic convergence cannot be expected (see Figure 5). On the other hand, the computational cost of the operator is negligible with respect to the DEM computation, and no perturbations are needed.

In the following, different numerical examples of UKO are shown for different stress and strain states (before and after peak, in the simulation of a biaxial test path). The coefficients obtained by the Kruyt method are higher in the diagonal than the ones obtained by the perturbation method. This is consistent with the fact that the upper bound extremum principle is used (Equations (14) and (15) vs Equation (9)) in the following.

UKO (prepeak):

$$
\underline{\underline{C}}^{\text {UКO }}=\left(\begin{array}{cccc}
1119.12 & -19.48 & 0 & 0 \\
-19.48 & 1119.56 & 0 & 0 \\
0 & 0 & 1119.12 & -19.48 \\
0 & 0 & -19.48 & 1119.56
\end{array}\right) \text {. }
$$

UKO (postpeak):

$$
\underline{\underline{C}}^{\text {UKO }}=\left(\begin{array}{cccc}
809.40 & 12.50 & 0 & 0 \\
12.50 & 1033.74 & 0 & 0 \\
0 & 0 & 809.40 & 12.50 \\
0 & 0 & 12.50 & 1033.74
\end{array}\right) .
$$

It can be seen in Equations (14) and (15) that, when applied to a small deformation gradient, those 2 matrices do not yield to a symmetric increment of the Cauchy stress tensor; for that, according to Equation (11), lines 2 and 3 of the matrices would have been identical. Anyway, that does not seem to be a problem because at each iteration of the Newton-Raphson process, the stress is calculated by the DEM computation and therefore is symmetric.

\section{2 | Upper bound corrected Kruyt operator}

Using statistical and thermodynamics principles, Kruyt shows that the elastic moduli can be obtained as the average of the upper and lower bounds if certain conditions are fulfilled..$^{22}$ The upper bound is used in the previous section (see Equation (13). Being aware of the linearity between upper and lower bounds and the actual elastic coefficients shown by Kruyt, the CTO in the prepeak part can be used to calibrate the UKO using a single coefficient $c_{x}$. This coefficient is then 

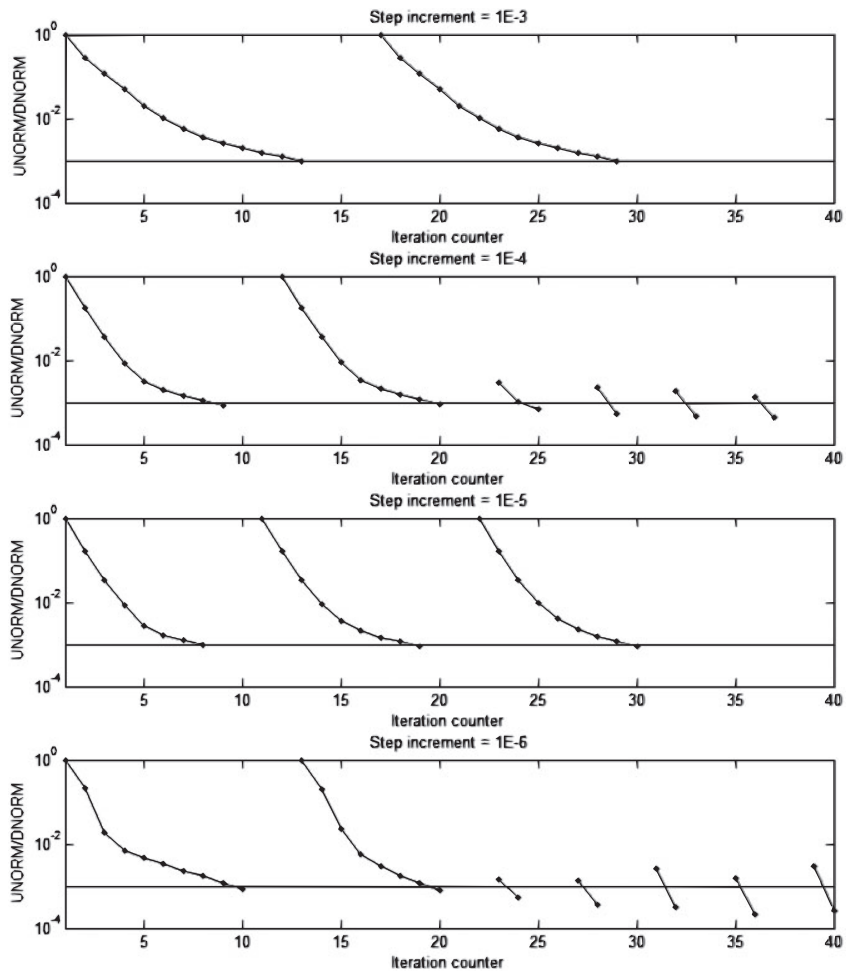

FIGURE 5 Displacement convergence profiles (UNORM/DNORM) for different initial step size: $\epsilon_{2}=1 \cdot 10^{-3}, \epsilon_{2}=1 \cdot 10^{-4}, \epsilon_{2}=1 \cdot 10^{-5}$, $\epsilon_{2}=1 \cdot 10^{-6}$. Kruyt operator. The horizontal line indicates the precision threshold

adopted in the whole test to obtain a better approximation of the elastic moduli: the UCKO. This operator has the same properties as the UKO with a slight increase of the convergence velocity as follows:

$$
C^{U С К O}=\frac{1}{\left|Y_{a}\right|} \sum_{c \in \mathcal{I}}\left(l^{c}\right)^{2} c_{x}\left(k_{n}\left(\vec{e}^{c} \otimes \vec{e}^{c}\right) \otimes\left(\vec{e}^{c} \otimes \vec{e}^{c}\right)+k_{t}\left(\vec{t}^{c} \otimes \vec{e}^{c}\right) \otimes\left(\vec{t}^{c} \otimes \vec{e}^{c}\right)\right),
$$

where $c_{x}$ is the calibrated coefficient.

A more complex calibration can be obtained by applying 2 calibrated coefficients: $c_{n}$ and $c_{t}$ to $k_{n}$ and $k_{t}$ in the expression (Equation (16)). These coefficients are calibrated using 2 terms of the CTO, ie, $C_{1111}$ and $C_{1122}$. This operator is called UCKO 2DOF (2-Degree-of-Freedom calibration), and it still provides a faster convergence in the prepeak close to quadratic velocity. Nevertheless, this does not improve the postpeak convergence velocity because the calibration using the CTO is done only once at the beginning of the test

$$
C^{\text {UCKO 2DOF }}=\frac{1}{\left|Y_{a}\right|} \sum_{c \in \mathcal{I}}\left(l^{c}\right)^{2}\left(c_{n} k_{n}\left(\vec{e}^{c} \otimes \vec{e}^{c}\right) \otimes\left(\vec{e}^{c} \otimes \vec{e}^{c}\right)+c_{t} k_{t}\left(\vec{t}^{c} \otimes \vec{e}^{c}\right) \otimes\left(\vec{t}^{c} \otimes \vec{e}^{c}\right)\right),
$$

where $c_{n}$ and $c_{t}$ are the correction coefficients.

\section{3 | Kruyt augmented operator}

The philosophy of the UKO "augmented" consists in the incorporation of additional DOF, ie, the rotation of the grains, as well as taking into account the preexisting forces. This is expected to provide a better physical representation of the equivalent elastic properties of the medium.

The operator "Kruyt" augmented $C^{K A O}$ is given at Equation (C.4), and in index notation, it reads

$$
\begin{aligned}
C_{i j k l}^{K A O}= & -\sigma_{i j} F_{l k}^{-1}+\sigma_{i h} F_{l h}^{-1} \delta_{j k} \\
& +\frac{1}{\left|Y_{a}\right|} \sum_{c \in \mathcal{I}}\left(l^{c}\right)^{2}\left(k_{n} e_{i}^{c} e_{j}^{c} e_{k}^{c} e_{h}^{c}+\frac{k_{t}}{2} t_{i}^{c} e_{j}^{c}\left(t_{k}^{c} e_{h}^{c}+e_{k}^{c} t_{h}^{c}\right)\right) F_{l h}^{-1} \\
& +\frac{1}{\left|Y_{a}\right|} \sum_{c \in \mathcal{I}} l^{c}\left(f_{n}^{c} t_{i}^{c}-f_{t}^{c} e_{i}^{c}\right) e_{j}^{c} t_{k}^{c} e_{h}^{c} F_{l h}^{-1} .
\end{aligned}
$$


A numerical example is presented as follows:

$$
\underline{\underline{C}}^{K A O}=\left(\begin{array}{cccc}
1117.9 & -10.2 & -10.2 & -0.1 \\
-20.5 & 559.4 & 559.0 & 0.0 \\
0.0 & 558.8 & 559.2 & -20.4 \\
-0.1 & -10.2 & -10.2 & 1118.3
\end{array}\right)
$$

Convergence is not as efficient as DEMQO/PSTLO (see Section 5): this is expected since this approach constitutes a rougher method of homogenization.

\section{5 | ELASTIC OPERATORS}

In this section, the periodic granular media is described, and different approaches to extracting operators are developed. The proposed operators are of elastic nature since no damage or grain sliding is considered in the developments. Two resulting operators are developed and tested: a DEMQO and a PSTLO.

\section{1 | DEM-based quasi-static operator}

A possible auxiliary operator is obtained considering the granular assembly as elastic in its current configuration. That consists in assuming that the contacts are persistent (neither loss nor creation of new ones) and that the grain interactions are elastic. The determination of the auxiliary operator can be performed numerically by DEM computations of 4 auxiliary steps for which the initial value of the macroscopic deformation gradient is $\boldsymbol{F}^{f}$ (that is to say, the value at the end of the current step) and the final values are $\boldsymbol{F}^{f}+\epsilon \boldsymbol{\Lambda}^{m n}, n, m=1,2$, where $\boldsymbol{\Lambda}^{m n}$ are of the form (5). Moreover, the friction coefficient among grains is set to a large value so as to prevent any sliding, thus insuring a quasi-elastic behavior. Naming $\mathrm{d} \boldsymbol{\sigma}^{\text {Enm }}$, the corresponding stress tensors are calculated at the end of these auxiliary steps, and the auxiliary operator (DEMQO) reads

$$
C_{i j m n}^{D E M Q O}=\frac{\mathrm{d} \sigma_{i j}^{E n m}}{\epsilon} .
$$

The DEM computational cost needed by the calculation of the DEMQO is almost negligible compared to the 4 complete DEM computations of the CTO evaluation. Consequently, the computational cost drops by an order of about 80\% (1 DEM calculation for the DEMQO compared to 5 for the CTO).

Despite being "elastic", it should be noted that the technique can still provide unstable results because bifurcation remains possible within the granular assembly.

Compared to the existing alternatives to be used in the FEM $\times$ DEM approach, notably the Kruyt operator, ${ }^{22}$ the here proposed elastic operator takes into consideration the microscale mechanics of the granular assembly and extract the elastic properties of the medium from the resolution of the microscale problem (either numerically or analytically, see Section 5.2), rather than performing a simplistic homogenization that does not consider the microscale mechanics. ${ }^{22}$

It is worth noting that strong conditions are usually applied to the DEM computations to guarantee a quasi-static state before the numerical homogenization. These conditions include a threshold for the kinetic energy, maximum number of sliding contacts, and maximum number of single contact grains. Such conditions force an extended relaxation period after the loading of the DEM assembly. The duration of this relaxation period is strongly dependent on the velocity of the applied deformation rather than the magnitude of this deformation; this has been observed in the testing of the DEMQO, showing relaxation times of the same order of magnitude both for the main DEM loading path and for the infinitesimal perturbations. In order to accelerate the code, quasi-static conditions have been made less strict for the perturbations, resulting in a drastic reduction of the computational time devoted to the perturbations. Notably, this does not affect the physics of the model ${ }^{\#}$ since the main loading path is still subject to strict

\footnotetext{
\# The perturbation calculations are not considered as part of the physics of the model because they merely provide the operator used in the Newton strategy but do not take part on the constitutive relation.
} 
quasi-static conditions. A numerical example using a compression biaxial test at the beginning of the loading is provided as follows:

$$
\underline{\underline{C}}^{\text {DEMQO }}=\left(\begin{array}{cccc}
902.3 & 13.4 & -14.2 & 86.2 \\
-13.4 & 384.6 & 421.9 & -12.4 \\
-13.4 & 384.6 & 421.9 & -12.4 \\
86.2 & -12.4 & -12.4 & 890.3
\end{array}\right)
$$

\section{2 | Prestressed truss-like operator}

The DEMQO proposed in the previous section is obtained through DEM computations in which everything is performed in such a way that the grain assembly behaves as far as possible elastically. The determination of the PSTLO follows the same idea. After the main DEM computation giving $S\left(\boldsymbol{F}^{f}\right)$ (see Equation (2)), the grain assembly is considered like a prestressed lattice with the rotation of the nodes as DOF. That means that the intergranular contacts are persistent and elastic. Submitted to a small increment $\mathrm{d} \boldsymbol{F}$ of the deformation gradient, the lattice-like granular assembly deforms from the stressed equilibrium configuration at the end of the DEM computation to another equilibrium configuration. The increment $\mathrm{d} \boldsymbol{F}$ being small, the displacements and the rotations of the grains are assumed small too, and the balance equations of the deformed configuration are linearized up to the first order with respect to the displacements $\vec{u}^{n}=\mathrm{d} \vec{x}^{n}$ and the rotations $\mathrm{d} \theta^{n}$ of the grains and of the contact forces $\mathrm{d} \vec{f}^{c}$. The linear problem to be solved to determine the PSTLO reads

$$
\begin{gathered}
\mathrm{d} \boldsymbol{F} \text { being given, find } \vec{u}^{n}, d \theta^{n} \text { and } \mathrm{d} \vec{f}^{c} \text { such that } \\
\begin{array}{r}
\forall \vec{u}^{n^{*}}, \forall \vec{\theta}^{n^{*}},-\sum_{c \in \mathcal{I}} \mathrm{d} \vec{f}^{c} \cdot\left(\vec{u}^{E(c) *}+\vec{\theta}^{E(c) *} \times \vec{a}^{c E}-\vec{u}^{O(c) *}-\vec{\theta}^{O(c) *} \times \vec{a}^{c O}\right) \\
+\sum_{c \in \mathcal{I}}\left(\left(\mathrm{d} \vec{a}^{c O} \times \vec{f}^{c}\right) \cdot \vec{\theta}^{O(c) *}-\left(\mathrm{d} \vec{a}^{c E} \times \vec{f}^{c}\right) \cdot \vec{\theta}^{E(c) *}\right)=0 \\
\mathrm{~d} \vec{a}^{c O}=\left(\alpha \vec{e}^{c} \otimes \vec{e}^{c}+\frac{l^{c O}}{l c}\left(\vec{t}^{c} \otimes \vec{t}^{c}\right)\right) \cdot \mathrm{d} \vec{b}^{c} \\
\mathrm{~d} \vec{a}^{c E}=-\left((1-\alpha) \vec{e}^{c} \otimes \vec{e}^{c}+\frac{l^{c E}}{l^{c}}\left(\vec{t}^{c} \otimes \vec{t}^{c}\right)\right) \cdot \mathrm{d} \vec{b}^{c} \\
\mathrm{~d} \vec{b}^{c}=\vec{u}^{E(c)}+\delta_{i}^{c} \mathrm{~d} \vec{Y}^{i}-\vec{u}^{O(c)} \\
\mathrm{d} \vec{Y}^{i}=\left(\mathrm{d} \boldsymbol{F} \cdot \boldsymbol{F}^{-1}\right) \cdot \vec{Y}^{i}, i=1,2 \\
\mathrm{~d} \vec{f}^{c}=\left(k_{n} \vec{e}^{c} \otimes \vec{e}^{c}+k_{t} \vec{t}^{c} \otimes \vec{t}^{c}+\frac{1}{l^{c}}\left(f_{n}^{c} \vec{t}^{c}-f_{t}^{c} \vec{e}^{c}\right) \otimes \vec{t}^{c}\right) \cdot \mathrm{d} \vec{b}^{c}-k_{t}\left(l^{c O} \mathrm{~d} \theta^{O(c)}+l^{c E} \mathrm{~d} \theta^{E(c)}\right) \vec{t}^{c} .
\end{array}
\end{gathered}
$$

The details of the notations and of the calculations leading to that formulation are given in Appendix B.

The solution of this problem yields to the contacts force $\mathrm{d} \vec{f}^{c}$ in terms of the increment $\mathrm{d} \boldsymbol{F}$, which, in turn, yield to the increment $d \sigma$ of the Cauchy stress tensor given by the expansion up to the first order of the Cauchy-Poisson formula as follows:

$$
\mathrm{d} \boldsymbol{\sigma}=\frac{1}{|Y|} \sum_{c \in \mathcal{I}} \delta_{i}^{c} \mathrm{~d} \vec{f}^{c} \otimes \vec{Y}^{i}+\boldsymbol{\sigma} \cdot \boldsymbol{F}^{-T} \cdot \mathrm{d} \boldsymbol{F}^{T}-\left(\boldsymbol{F}^{-T}: \mathrm{d} \boldsymbol{F}\right) \boldsymbol{\sigma} .
$$

Denoting $\mathrm{d} \boldsymbol{\sigma}^{n m}$ the increments of the stress tensors corresponding to the increments $\mathrm{d} \boldsymbol{F}=\boldsymbol{\Lambda}^{m n}, n, m=1,2$, where the $\Lambda^{m n}$ are of the form (5), the auxiliary operator (PSTLO) reads

$$
C_{i j m n}^{P S T L O}=\mathrm{d} \sigma_{i j}^{n m} .
$$

An analytic operator is therefore obtained, thus avoiding the need to run the numerical DEM: this can reduce the computational charge without any compromise in the results. 
Indeed, beside the numerical precision, this approach provides the same results as the DEMQO, as it needs to solve a linear system of equations. However, it is worth noting that the solution of a linear system requires a computational time with cubic dependence on the size of the system: the numerical efficiency of the PSTLO over the DEMQO then depends on the complexity of the microscale model.

\section{6 | RESULTS}

In Figure 6, the convergence profiles of the previously defined compression biaxial test (see Figure 2) are presented. The 2 plots represent the evolution of the residual force vector in the initial stage of the compression (see Figure 6-left) and well after the stress peak (see Figure 6-right). The case at the beginning of the compression (Figure 6-left) gives the best convergence rates for the CTO, DEMQO/PSTLO, and UCKO 2DOF in this order for a threshold" FNORM/RNORM= $1 \cdot 10^{-2}$. It is also interesting to observe the asymptotic value around $F N O R M / R N O R M=3 \cdot 10^{-4}$ due to the precision of the constitutive law. The plot for the postpeak regime (Figure 6-right) gives the best convergence for UCKO, DEMQO/PSTLO, and CTO in this order for a threshold FNORM/RNORM $=1 \cdot 10^{-2}$. In this case, the asymptotic value for the precision is around $2 \cdot 10^{-4}$ due to the discrete nature of the DEM and the presence of grain sliding.

A second plot (see Figure 7-left) shows the convergence performance of the different operators, focusing on the number of iterations needed to reach convergence with a precision threshold FNORM/RNORM $=1 \cdot 10^{-2}$. It shows that the best overall performance is obtained with DEMQO/PSTLO. In the case of the UCKO and UCKO 2DOF operators, it can be seen that the calibration improves the performance in the initial stages of the biaxial loading, which is consistent with the fact that the proposed calibration is performed with reference to the CTO at the beginning of the loading (CTO being nonavailable later). It can be observed that the prepeak phase needs more iterations than the postpeak. This is most likely due to the perfect initial homogeneity of the problem both at the micro and at the macroscale. This point has been addressed in a previous paper (see the work of Shahin et $\mathrm{al}^{12}$ ), in which the effect of the initial heterogeneity has been studied by using a random distribution of equivalent but different REVs at the microscale. Table 1 summarizes the qualities and convergence velocity ${ }^{* *}$ of the different operators, and references are provided for the operators developed outside this work (CTO, AEO, and UKO).

The performances of the operators are compared in terms of relative wall-clock time of the compression biaxial test simulation till $2 \%$ of axial deformation. The CTO/AEO operator (only AEO is used in the postpeak regime) is here considered as the reference result, and the related wall-clock is normalized to 100 (see Figure 7-right). The PSTLO

" FNORM is the norm of the nodal forces and RNORM of the nodal reactions in the current iteration.

${ }^{* *}$ The convergence velocity is computed as the inverse of the number of integrations of the loading path, both for the initial stages $\left(V_{i}\right)$ and postpeak $\left(V_{p}\right)$, and the values are normalized with the reference of the DEMQO/PSTLO. Note that in the perturbation-based methods, the number of needed integrations is 5 for each iteration.
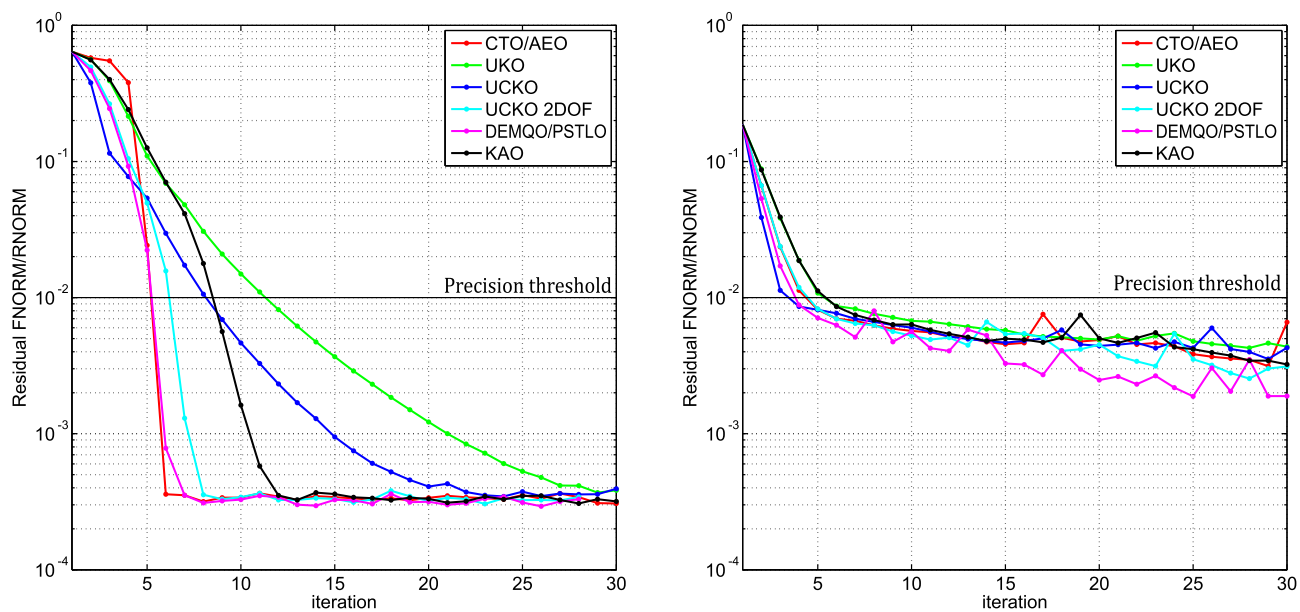

FIGURE 6 Convergence plots of the tested operators; left: at the beginning of a biaxial test with $\epsilon_{22}=0$; right: postpeak regime with $\epsilon_{22}=1.5 \%$. $y$-axis in logarithmic scale. AEO, auxiliary elastic operator; CTO, consistent tangent operator; DEMQO, DEM-based quasi-static operator; KAO, Kruyt augmented operator; PSTLO, prestressed truss-like operator; UCKO, upper bound corrected Kruyt operator; UKO 2DOF, upper bound corrected Kruyt operator with 2 degrees of freedom [Colour figure can be viewed at wileyonlinelibrary.com] 

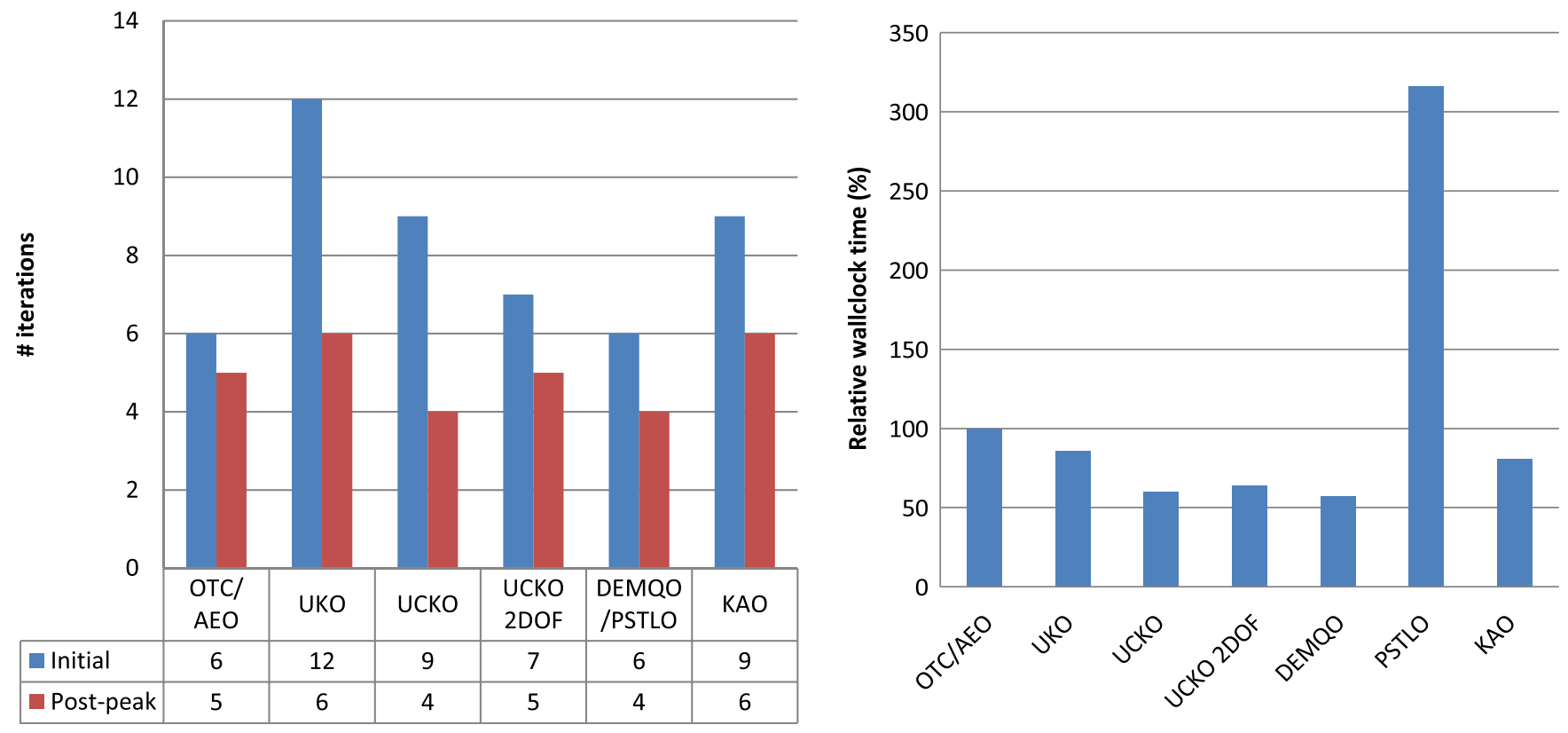

FIGURE 7 Left: number of iterations needed to reach convergence with the different operators. Convergence threshold: FNORM/RNORM = $1 \cdot 10^{-2}$. Right: relative wall-clock time for a biaxial loading till $2 \%$ of axial deformation. Processor: Intel ${ }^{\circledR}$ Core i5-6440HQ @2.60 GHz [Colour figure can be viewed at wileyonlinelibrary.com]

TABLE 1 Summary operators

\begin{tabular}{lllccc} 
Operator & Advantages & Disadvantages & $\boldsymbol{V}_{\boldsymbol{i}}$ & $\boldsymbol{V}_{\boldsymbol{p}}$ & References \\
\hline CTO & Quadratic & Perturbations & 0.20 & - & eq. 6 in the work of Simo and Taylor ${ }^{23}$ \\
AEO & No perturbations & Not updated & 0.20 & 0.80 & Nguyen $^{10}$ \\
DEMQO & Robust, fast & Numerical & 1.00 & 1.00 & Equation (20) \\
PSTLO & Robust, fast & System solver & 1.00 & 1.00 & Appendix B \\
UKO & Simple computation & Not physical & 0.50 & 0.67 & eq. 13 in the work of Kruyt and Rothenburg \\
UCKO & Better fit & Not physical & 0.67 & 1.00 & Equation (16) \\
UCKO 2DOF & Yet better fit & Not physical & 0.86 & 0.80 & Equation (17) \\
KAO & Considers rotations & Not physical & 0.67 & 0.67 & Appendix C \\
\hline
\end{tabular}

Abbreviations: AEO, auxiliary elastic operator; CTO, consistent tangent operator; DEMQO, DEM-based quasi-static operator; KAO, Kruyt augmented operator; PSTLO, prestressed truss-like operator; UCKO, upper bound corrected Kruyt operator; UKO 2DOF, upper bound corrected Kruyt operator with 2 degrees of freedom.

presents the highest computational time due to the previously discussed linear system resolution in the microscale. The total wall-clock time, ie, 3 times higher than the any other operator, makes the PSTLO not appropriate for a 400-particle microscale: its use is reserved for problems with, at most, a 100-particle microscale. As already pointed out, in addition, the CTO/AEO is far less efficient than the other operators due to the time needed to integrate the perturbations along the loading path. The Kruyt-type operators are at least $10 \%$ more efficient than the reference solution. The calibrated operators are about $25 \%$ faster than the standard UKO. However, the UCKO-like operators require a calibration phase that is not here considered and that can be hardly extended to different problems. The KAO, which accounts for rotations preexisting forces in the microscale, represents an enhancement of the original UKO. Finally, it is worth noting that the DEMQO offers the most interesting performances, both in terms of the number of iterations and the total wall-clock time. The here proposed approach is indeed 35\% more efficient than the commonly adopted Kruyt operator.

\section{7 | PARALLELIZATION}

The model takes advantage of an element loop parallelization implemented using an OpenMP paradigm. This parallelization allows to significantly reduce the total wall-clock time of the simulation, and the speedup is almost proportional to the number of used cores. 
TABLE 2 Time spent on the different parts of the FEM model for 2 biaxial tests FEM $\times$ DEM with 128 and 512, respectively

\begin{tabular}{lcc} 
Wall-Clock Time (\%) & 128 Elements & 512 Elements \\
\hline Elements & 99.93 & 99.67 \\
Solver & 0.02 & 0.31 \\
Other & 0.05 & 0.02 \\
Total & 100 & 100 \\
\hline
\end{tabular}
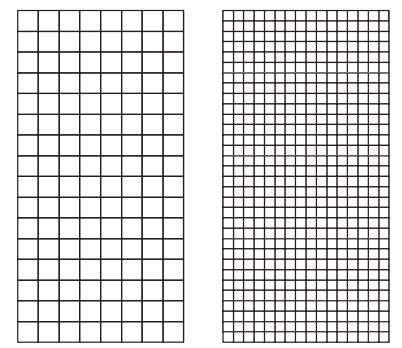

FIGURE 8 Biaxial test geometry with 128 and 512 elements

\section{1 | Element loop vs solver speedup}

FEM can be parallelized both in the Gauss point integration loop (or element loop) and the solver part. Typically, problems with analytic laws and fine meshes are solver intensive, meaning, that most of the computation time is spent on the solver. On the other hand, double scale problems with coarse macroscale mesh are element intensive, meaning that most of the computation time is spent on the integration of the Gauss points in the element loop. This implies, according to Amdahl, ${ }^{24}$ a theoretical possible speedup given as

$$
S(n)=\frac{T(1)}{T(n)}=\frac{1}{B+\frac{1}{n}(1-B)},
$$

where $S$ is the speedup, $n$ the number of parallel regions, and $B$ the nonparallelizable part of the code translated to CPU time. The element loop parallelization is less and less efficient as we increase the number of elements because the time devoted to the solver increases super linearly with the size of the global stiffness matrix. ${ }^{25}$ On the other hand, for a large number of cores involved in the parallelization, the speedup factor tends to an asymptotic value linked to the time devoted to the nonparallelizable part (see Equation (24)). This 2 factors set the upper limits to the effectiveness of an element loop parallelization. ${ }^{\dagger \dagger}$

In this present work, consisting in $\mathrm{FEM} \times \mathrm{DEM}$, the situation is clearly element intensive (see Table 2), and it consists in the same configuration used in the operator study, ie, compression biaxial test, 4 Gauss points per element, microscale with 400 spherical particles, frictional-cohesive contacts, 3 mesh refinements are used in the following: 128 and 512 elements (see Figure 8). Therefore, an element parallelization is implemented. It consists in an OpenMP implementation (shared memory paradigm), which executes concurrent element computations using different processor cores. The concern is to avoid concurrency in the global stiffness matrix assembly, and this is done using the "atomic" construct in the OpenMP implementation: !\$OMP ATOMIC, it ensures that a specific storage location is updated automatically, rather than exposing it to the possibility of multiple simultaneous writing threads. ${ }^{26}$

\section{2 $\mid$ Performance}

The parallelized code is executed in different machines to benchmark its performances. The machines are 2 servers, ie, one with 2 Intel ${ }^{\circledast}$ Xeon $^{\circledR}$ CPU E5410 @2.33-GHz 4-core processor (8 cores in total) and the other one with 2 Intel ${ }^{\circledR}$ Xeon $^{\circledR}$ CPU E5650 @2.67-GHz 6-core processor (12 cores in total). A standard 512-element simulation consisting in a compression biaxial test till $2 \%$ of axial deformation is executed both sequentially and parallelized in the 2 machines. The final value of $2 \%$ of axial deformation is chosen to enter the postpeak region in which the computational charge per iteration is much

${ }^{\dagger \dagger}$ As an example, for less than 512 elements, the speedup presents an almost linear relation with the number of processors if this number is less than 50 (parallelization efficiency equal to $86 \%$ with a 512-element test and 50 available cores). 


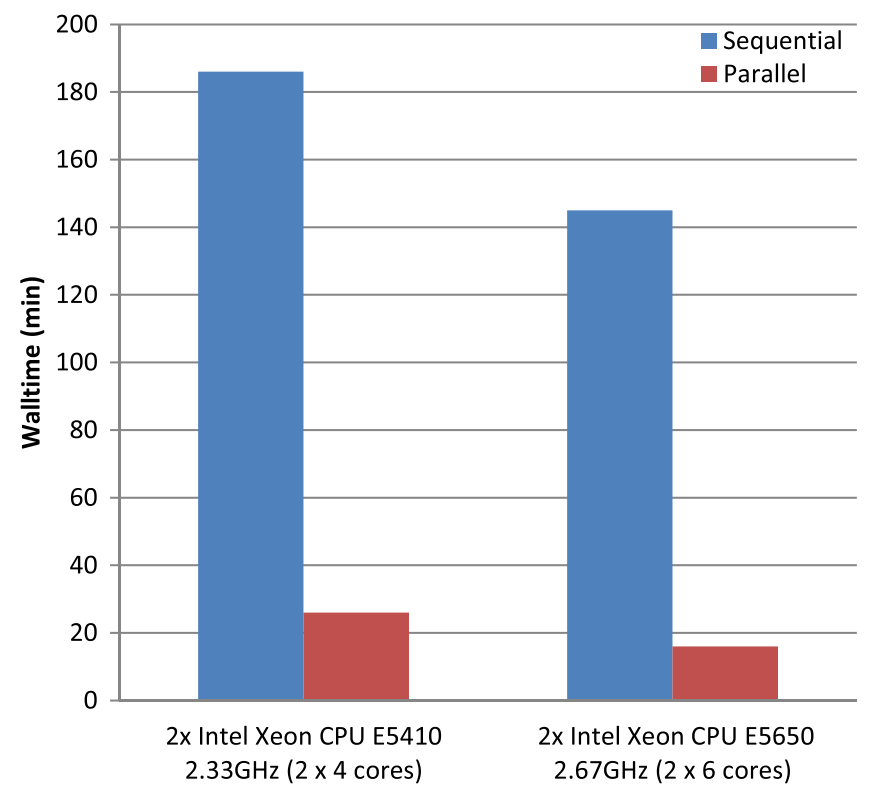

FIGURE 9 Parallelization performance: comparison of sequential and parallel wall-clock time of a FEM $\times$ DEM simulation in different machines [Colour figure can be viewed at wileyonlinelibrary.com]

larger than in the prepeak region. The results show a speedup of 7.15 for the machine with 8 cores and 9.06 for the machine with 12 cores (see Figure 9). The good efficiency of the parallelization proves the aforementioned features of the model.

\section{8 | CONCLUSIONS}

A FEM $\times$ DEM model has been presented; this approach overcomes the phenomenological assumptions and calibrations needed in a classical analytic law used in FEM. Given the inefficacy of the CTO in the postpeak range, a variety of Newton's operators has been reviewed and proposed to study and improve the stability and numerical efficiency of the method. The AEO is a first attempt to build a stable operator by averaging several CTOs. Kruyt derived operators bring yet an improvement of the convergence and stability. The KAO shows that an enrichment of the physical description of a Kruyt-type operator can further improve the operator performance. The DEMQO and PSTLO, which treat the DEM assembly as a prestressed elastic truss, give the best results in terms of the number of iterations both in the prepeak and postpeak regimes, becoming good candidates to be used in the FEM $\times$ DEM approach. It is worth noting that these 2 operators perform very differently in terms of wall-clock time. Indeed, the DEMQO operator turns out to be the most efficient operator, overtopping the existing approaches.

An element loop parallelization has also been developed, thus reducing the overhead due to sequential element calculations and resulting in a major speedup of the simulations.

Further developments are needed to ensure a proper shear band description, ie, regularization is required for reducing the pathological mesh dependency when strain localization occurs. The model can be further improved by parallelizing the solver and by implementing a massive parallelization, ie, Message-Passing Interface (MPI), changing the parallelization paradigm from the shared memory one used here (OpenMP) to a distributed memory one (MPI), which allows to use larger numbers of cores.

\section{ORCID}
A. Argilaga (D) http://orcid.org/0000-0002-2812-9258
S. Dal Pont (i) http://orcid.org/0000-0002-0348-5918

\section{REFERENCES}

1. Auriault J. Heterogeneous medium. Is an equivalent macroscopic description possible? Int J Eng Sci. 1991;29(7):785-795.

2. Smit R, Brekelmans W, Meijer H. Prediction of the mechanical behavior of nonlinear heterogeneous systems by multi-level finite element modeling. Comput Methods Appl Mech Eng. 1998;155(1):181-192. 
3. Kouznetsova V, Brekelmans W, Baaijens F. An approach to micro-macro modeling of heterogeneous materials. Comput Mech. 2001;27(1):37-48.

4. Auriault J-L. Heterogeneous periodic and random media. Are the equivalent macroscopic descriptions similar? Int $J$ Eng Sci. 2011;49(8):806-808.

5. Hautefeuille M, Colliat J-B, Ibrahimbegovic A, Matthies H, Villon P. A multi-scale approach to model localized failure with softening. Comput Struct. 2012;94:83-95.

6. Kaneko K, Terada K, Kyoya T, Kishino Y. Global-local analysis of granular media in quasi-static equilibrium. Int J Solids Struct. 2003;40(15):4043-4069.

7. Miehe C, Dettmar J, Zäh D. Homogenization and two-scale simulations of granular materials for different microstructural constraints. Int J Numer Methods Eng. 2010;83(8-9):1206-1236.

8. Nitka M, Combe G, Dascalu C, Desrues J. Two-scale modeling of granular materials: a DEM-FEM approach. Granul Matter. 2011;13(3):277-281.

9. Guo N, Zhao J. A hierarchical model for cross-scale simulation of granular media. AIP Conf Proc. 2013;9(11):789-818.

10. Nguyen T. Modélisation numérique à double échelle des matériaux granulaires cohésifs: approche par éléments finis-éléments discrets PhD thesis. 2013.

11. Nguyen T, Combe G, Caillerie D, Desrues J. FEM $\times$ DEM modelling of cohesive granular materials: numerical homogenisation and multi-scale simulations. Acta Geophysica. 2014;62(5):1109-1126.

12. Shahin G, Desrues J, Dal Pont $S$, Combe G, Argilaga A. A study of the influence of REV variability in double scale FEM $\times$ DEM analysis. Int J Numer Methods Eng. 2016;107(10):136-144.

13. Guo N, Zhao J. A coupled FEM/DEM approach for hierarchical multiscale modelling of granular media. Int J Numer Methods Eng. 2014;1542:1222-1225.

14. Guo N, Zhao J. Multiscale insights into classical geomechanics problems. Int J Numer Anal Methods Geomech. 2015a;40(3):367-390.

15. Liu Y, Sun W, Yuan Z, Fish J. A nonlocal multiscale discrete-continuum model for predicting mechanical behavior of granular materials. Int J Numer Methods Eng. 2015;106(2):129-160.

16. Wang K, Sun W. A semi-implicit discrete-continuum coupling method for porous media based on the effective stress principle at finite strain. Comput Methods Appl Mech Eng. 2016;304:546-583.

17. Guo N, Zhao J. Parallel hierarchical multiscale modelling of hydro-mechanical problems for saturated granular soils. Comput Methods Appl Mech Eng. 2016b;305:37-61.

18. Guo N, Zhao J. 3D multiscale modeling of strain localization in granular media. Comput Geotechn. 2016;80:360-372.

19. Mokni M, Desrues J. Strain localization measurements in undrained plane-strain biaxial tests on Hostun RF sand. 1999;4(4):419-441.

20. Guo N, Zhao J. A multiscale investigation of strain localization in cohesionless sand. In: Bifurcation and Degradation of Geomaterials in the New Millennium. Berlin, Germany: Springer; 2015:121-126.

21. Pasternak E, Dyskin AV, Sevel G. Chains of oscillators with negative stiffness elements. J Sound Vib. 2014;333(24):6676-87.

22. Kruyt N, Rothenburg L. Statistical theories for the elastic moduli of two-dimensional assemblies of granular materials. Int J Eng Sci. 1998;36(10):1127-1142.

23. Simo JC, Taylor RL. Consistent tangent operators for rate-independent elastoplasticity. Comput Methods Appl Mech Eng. 1985;48(1):101-118.

24. Amdahl GM. Validity of the single processor approach to achieving large scale computing capabilities. Paper presented at: Proceedings of the Spring Joint Computer Conference; April 18-20, 1967; Atlantic City, NJ.

25. Golub GH, Van Loan CF. Matrix Computations. Vol. 3. Baltimore, MD: John Hopkins University Press; 2012:94-103.

26. OpenMP Architecture Review Board. OpenMP application program interface, version 3.1. 2011. http://www.openmp.org

How to cite this article: Argilaga A, Desrues J, Dal Pont S, Combe G, Caillerie D. FEM $\times$ DEM multiscale modeling: Model performance enhancement from Newton strategy to element loop parallelization. Int J Numer Methods Eng. 2017;1-19. https://doi.org/10.1002/nme.5732

\section{APPENDIX A : DESCRIPTION OF THE PERIODIC GRANULAR ASSEMBLY}

\section{A.1 Numberings and geometry}

This first part of the appendix is intended to make precise the notations used in the main part of this paper and in the following of the appendix.

The grains are 2D disks; the whole study is limited to 2 dimensions. The grains move in a 2D plane orthogonal to the normed vector $\vec{i}_{3}$ that orients the plane. 


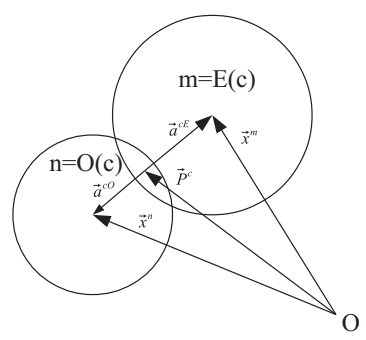

FIGURE A1 Two circular grains in contact

Conditions of periodicity are used, which means that the sample of grains is considered as the base cell of a periodic medium, and the vectors of periodicity depend on the time and are denoted as $\vec{Y}^{1}$ and $\vec{Y}^{2}$. A grain of the base cell can then interact with another grain of that cell of a neighboring cell.

The grains of the base cell are numbered $n, m, \ldots$. Let $R^{n}$ denote the radius of the grain $n$. The position of the center of the grain $n$ is given with respect to an origin $O$ by the vector $\vec{x}^{n}\left(\vec{x}^{n}\right.$ depends on the time).

A contact $c=\left(n, m, \delta_{1}, \delta_{2}\right)$ is an ordered couple of grains $n$ and $m$ and, as conditions of periodicity are used, 2 numbers $\delta_{1}$ and $\delta_{2}$, taking values in $\{-1,0 ; 1\}$ that tell whether the contact is between grains of the base cell or between grains of 2 neighboring cells. The set of all contacts is denoted as $\mathcal{I}$. The couple of grains of the contact $c$ being ordered, one, say $n$, is called the origin $O(c)$ of the contact and the other $m$ is the end $E(c)$. The $2 \delta^{\prime}$ s are denoted as $\delta_{1}^{c}$ and $\delta_{2}^{c}$ (see Figure A1). The branch vector of the contact $c$ is the vector $\vec{b}^{c}$ linking the centers of the 2 grains in contact as follows:

$$
\vec{b}^{c}=\vec{x}^{E(c)}+\delta_{1}^{c} \vec{Y}^{1}+\delta_{2}^{c} \vec{Y}^{2}-\vec{x}^{O(c)} .
$$

In the following, according to the Einstein's summation convention, the sum $\delta_{1}^{c} \vec{Y}^{1}+\delta_{2}^{c} \vec{Y}^{2}$ is simplified into $\delta_{i}^{c} \vec{Y}^{i}$. Let $\vec{e}^{c}$ and $l^{c}$ be the unit vector and the length of the branch vector $\vec{b}^{c}: \vec{b}^{c}=l^{c} \vec{e}^{c}, 0<l^{c},\left\|\vec{e}^{c}=1\right\| \cdot \vec{e}^{c}$ is the normal vector to the contact, and let $\vec{t}^{c}$ denote the tangent vector image of $\vec{e}^{c}$ by the counterclockwise rotation of angle $\pi / 2$.

In the considered modeling of the contacts, grains can overlap, let

$$
h^{c}=R^{O(c)}+R^{E(c)}-l^{c}
$$

be the overlap of the contact $c$. As grains in contact can overlap, it is necessary to be able to write balance equations for moments to define a contact point, the position of which is given by the vector $\vec{p}^{c}$. Let $\vec{a}^{c O}$ and $\vec{a}^{c E}$ be the vectors: $\vec{a}^{c O}=\vec{x}^{O(c)}-\vec{p}^{c}$ and $\vec{a}^{c E}=\vec{x}^{E(c)}-\vec{p}^{c}$. The contact point is chosen on the segment linking the centers of the 2 grains, which entails that the lever arms $\vec{a}^{c O}$ and $\vec{a}^{c E}$ are parallel to $\vec{e}^{c}$ as follows:

$$
\begin{aligned}
\vec{a}^{c O} & =l^{c O} \vec{e}^{c} \\
\vec{a}^{c E} & =-l^{c E} \vec{e}^{c}
\end{aligned}
$$

and obviously,

$$
l^{c O}+l^{c E}=l^{c} .
$$

When 2 grains in contact overlap $\left(h^{c}>0\right)$, it seems sensible to impose that the contact point should be in the overlap region that reads

$$
l^{c O}=R^{O(c)}-\alpha h^{c},
$$

where $\alpha$ is a parameter such that $0 \leq \alpha \leq 1$, for instance, $\alpha=1 / 2$. From (A.4) and (A.2), it comes

$$
l^{c E}=R^{E(c)}-(1-\alpha) h^{c} .
$$

\section{A.2 Contact forces and equilibrium}

Grains in contact interact by shear forces, which means that the rolling torque is disregarded. For a contact $c$, let $\vec{f}^{c}$ be the force applied by the grain $E(c)$ on $O(c)$, as the rolling couple is disregarded the moment at the center $\vec{x}^{n}$ of the force $\vec{f}^{c}$ is $\vec{a}^{c O} \times \vec{f}^{c}$. 
The equilibrium of the whole granular assembly reads in a virtual power formulation as follows:

$$
\forall \vec{u}^{n *}, \forall \vec{\theta}^{n *},-\sum_{c \in I}\left[\vec{f}^{c} \cdot\left(\vec{u}^{E(c) *}-\vec{u}^{O(c) *}\right)+\left(\vec{a}^{c E} \times \vec{f}^{c}\right) \cdot \vec{\theta}^{E(c) *}-\left(\vec{a}^{c O} \times \vec{f}^{c}\right) \cdot \vec{\theta}^{O(c) *}\right]=0 .
$$

In that formulation, the virtual rotation vectors are $\vec{\theta}^{n *}=\theta^{n *} \vec{i}_{3}$, in which $\vec{i}_{3}$ is the normed vector orthogonal to the plane where the 2D grains move.

\section{APPENDIX B : DETERMINATION OF PSTLO}

With the configuration of the base cell being determined by the 2 periodicity vectors $\vec{Y}^{1}$ and $\vec{Y}^{2}$, the positions of the grains, and the contact forces $\vec{f}^{c}$, an increment $\mathrm{d} \boldsymbol{F}$ is added to the macroscopic deformation gradient $\boldsymbol{F}$, the PSTLO is the operator that yields the increment $\mathrm{d} \sigma$ of the Cauchy stress $\sigma$ in terms of $\mathrm{d} \boldsymbol{F}$. The increment $\mathrm{d} \boldsymbol{F}$ yields increments $\mathrm{d} \vec{Y}^{1}$ and $\mathrm{d} \vec{Y}^{2}$ of the vectors $\vec{Y}^{1}$ and $\vec{Y}^{2}$ given as

$$
\mathrm{d} \vec{Y}^{i}=\left(\mathrm{d} \boldsymbol{F} \cdot \boldsymbol{F}^{-1}\right) \cdot \vec{Y}^{i}, i=1,2,
$$

where $\mathrm{d} \boldsymbol{F} \cdot \boldsymbol{F}^{-1}$ denotes the composition product of $\mathrm{d} \boldsymbol{F}$ and $\boldsymbol{F}^{-1}$ and $\left(\mathrm{d} \boldsymbol{F} \cdot \boldsymbol{F}^{-1}\right) \cdot \vec{Y}^{i}$ denotes the image of $\vec{Y}^{i}$ by the linear application $\mathrm{d} \boldsymbol{F} \cdot \boldsymbol{F}^{-1} \cdot \vec{Y}_{R}^{1}=F^{-1} \cdot \vec{Y}^{1}$ and $\vec{Y}_{R}^{2}=F^{-1} \cdot \vec{Y}^{2}$ are the periodicity vectors of the initial configuration of the base cell. According to (A.1), the changes of $\vec{Y}^{1}$ and $\vec{Y}^{2}$ at least induce changes in the distances between grains and consequently changes $\mathrm{d} \vec{f}^{c}$ in the contact forces. Consequently, the balance of the grains is broken and they have to move and rotate to recover the equilibrium.

As the increment $\mathrm{d} \boldsymbol{F}$ is assumed small, the resulting displacements $\vec{u}^{n}=\mathrm{d} \vec{x}^{n}$ and rotations $\mathrm{d} \theta^{n}$ of the grains are small, and it is possible to develop the geometric quantities, the forces, and the equations of equilibrium up to the first order to define the linear problem to be solved to determine $\vec{u}^{n}, \mathrm{~d} \theta^{n}$ in terms of $\mathrm{d} \boldsymbol{F}$, which will yield $\mathrm{d} \vec{f}^{c}$ in terms of $\mathrm{d} \boldsymbol{F}$ and consequently the increment $\mathrm{d} \sigma$ of the Cauchy stress $\sigma$ in terms of $\mathrm{d} \boldsymbol{F}$, hence defining the looked-for operator.

\section{B.1 | Incremental balance equation}

The differentiation of the virtual power formulation (A.7) of the balance equation reads

$$
\begin{aligned}
\forall \vec{u}^{n *}, \forall \vec{\theta}^{n *}, & -\sum_{c \in \mathcal{I}} \mathrm{d} \vec{f}^{c} \cdot\left(\vec{u}^{E(c) *}+\vec{\theta}^{E(c) *} \times \vec{a}^{c E}-\vec{u}^{O(c) *}-\vec{\theta}^{O(c) *} \times \vec{a}^{c O}\right) \\
& +\sum_{c \in \mathcal{I}}\left(\left(\mathrm{d} \vec{a}^{c O} \times \vec{f}^{c}\right) \cdot \vec{\theta}^{O(c) *}-\left(\mathrm{d} \vec{a}^{c E} \times \vec{f}^{c}\right) \cdot \vec{\theta}^{E(c) *}\right)=0,
\end{aligned}
$$

where $\mathrm{d} \vec{a}^{C O}$ and $\mathrm{d} \vec{a}^{c E}$ are the increments of the lever arms $\vec{a}^{c O}$ and $\vec{a}^{c E}$.

\section{B.2 | Differentiation of the geometry}

In the motion of the grains resulting from the change of $\boldsymbol{F}$ into $\boldsymbol{F}+\mathrm{d} \boldsymbol{F}$, the positions of the grains change yielding the changes in $\vec{b}^{c}, \vec{e}^{c}, \vec{t}^{c}$, and $h^{c}$, and it is necessary to determine those changes, ie, $\mathrm{d} \vec{b}^{c}$, $\mathrm{d} \vec{e}^{c}, \mathrm{~d} \vec{t}^{c}$, and $\mathrm{d} h^{c}$, that is assumed to be small in terms of the displacements $\vec{u}^{n}$ and rotations $\mathrm{d} \theta^{n}$ of the grains.

\section{B.3 Differentiation of $\vec{b}^{c}, \vec{e}^{c}, \vec{t}^{c}$, and $h^{c}$}

The differentiation of (A.1) with respect to $\vec{u}^{n}$ and $\mathrm{d} \theta^{n}$ yields

$$
\mathrm{d} \vec{b}^{c}=\vec{u}^{E(c)}+\delta_{i}^{c} \mathrm{~d} \vec{Y}^{i}-\vec{u}^{O(c)} .
$$

The differentiation of $\vec{b}^{c}=l^{c} \vec{e}^{c}$ yields $\mathrm{d} \vec{b}^{c}=\mathrm{d} l^{c} \vec{e}^{c}+l^{c} \mathrm{~d} \vec{e}^{c}$. Taking into account that $\vec{e}^{c}$ is a norm vector and consequently that $\vec{e}^{c} \cdot \mathrm{d} \vec{e}^{c}=0$ and that the problem is $2 \mathrm{D}$,

$$
\begin{aligned}
\mathrm{d} \vec{e}^{c} & =\frac{1}{l^{c}}\left(\vec{t}^{c} \otimes \vec{t}^{c}\right) \mathrm{d} \vec{b}^{c} \\
\mathrm{~d} \vec{t}^{c} & =-\frac{1}{l^{c}}\left(\vec{e}^{c} \otimes \vec{t}^{c}\right) \cdot \mathrm{d} \vec{b}^{c} .
\end{aligned}
$$

The differentiation of (A.2) yields

$$
\mathrm{d} h^{c}=-\mathrm{d} l^{c}=-\vec{e}^{c} \cdot \mathrm{d} \vec{b}^{c}
$$




\section{B.4 | Differentiation of the lever arms $\vec{a}^{c O}$ and $\vec{a}^{c E}$}

Taking into account (B.5), the differentiation of (A.3), (A.5), and (A.6), yields after some algebra that is given as

$$
\begin{aligned}
\mathrm{d} \vec{a}^{c O} & =\left(\alpha \vec{e}^{c} \otimes \vec{e}^{c}+\frac{l^{c O}}{l^{c}}\left(\vec{t}^{c} \otimes \vec{t}^{c}\right)\right) \cdot \mathrm{d} \vec{b}^{c} \\
\mathrm{~d} \vec{a}^{c E} & =-\left((1-\alpha) \vec{e}^{c} \otimes \vec{e}^{c}+\frac{l^{c E}}{l^{c}}\left(\vec{t}^{c} \otimes \vec{t}^{c}\right)\right) \cdot \mathrm{d} \vec{b}^{c} .
\end{aligned}
$$

\section{B.5 | Incremental forces and moments}

The contact force $\vec{f}^{c}$ can be decomposed into the following:

$$
\vec{f}^{c}=f_{n}^{c} \vec{e}^{c}+f_{t}^{c} \vec{t}^{c}
$$

the differentiation of which reads

$$
\mathrm{d} \vec{f}^{c}=\mathrm{d} f_{n}^{c} \vec{e}^{c}+f_{n}^{c} \mathrm{~d} \vec{e}^{c}+\mathrm{d} f_{t}^{c_{t}^{c}} \vec{t}^{c}+f_{t}^{c} \mathrm{~d} \vec{t}^{c} .
$$

The constitutive equation of the normal component $f_{n}^{c}$ being $f_{n}^{c}=-k_{n}\left(h^{c}-C\right)$ comes

$$
\mathrm{d} f_{n}^{c}=-k_{n} \mathrm{~d} h^{c},
$$

that is to say, according to (B.5), we have

$$
\mathrm{d} f_{n}^{c}=k_{n} \vec{e}^{c} \cdot \mathrm{d} \vec{b}^{c}
$$

The constitutive equation of the tangent component $\mathrm{d} f_{t}^{c}$ reads

$$
\mathrm{d} f_{t}^{c}=k_{t}\left(\mathrm{~d} \vec{b}^{c} \cdot \vec{t}^{c}-\left(l^{c O} \mathrm{~d} \theta^{O(c)}+l^{c E} \mathrm{~d} \theta^{E(c)}\right)\right) .
$$

Taking into account Equations (B.4a), (B.4b), (B.8), (B.9), and (B.10), the force increment $\mathrm{d} \vec{f}^{c}$ reads

$$
\mathrm{d} \vec{f}^{c}=\left(k_{n} \vec{e}^{c} \otimes \vec{e}^{c}+k_{t} \vec{t}^{c} \otimes \vec{t}^{c}+\frac{1}{l^{c}}\left(f_{n}^{c} \vec{t}^{c}-f_{t}^{c} \vec{e}^{c}\right) \otimes \vec{t}^{c}\right) \cdot \mathrm{d} \vec{b}^{c}-k_{t}\left(l^{c O} \mathrm{~d} \theta^{O(c)}+l^{c E} \mathrm{~d} \theta^{E(c)}\right) \vec{t}^{c} .
$$

\section{B.6 Determination of $d \sigma$ in terms of $d F$}

The vectors $\vec{Y}^{1}$ and $\vec{Y}^{2}$ defining the cell, the positions $\vec{x}^{n}$ of the grains, the set $\mathcal{C}$ of all the contacts, the contact forces $\vec{f}^{c}$, the macroscopic deformation gradient $F$ being known and the increment $\mathrm{d} \boldsymbol{F}$ of $\boldsymbol{F}$ being given, the displacements $\vec{u}^{n}$ and the rotations $\mathrm{d} \theta^{n}$ of the grains, and the increments $\mathrm{d} \vec{f}^{c}$ of the contact forces are solutions of the problem (22). The displacements $\vec{u}^{n}$, the rotations $\mathrm{d} \theta^{n}$, and the increments $\mathrm{d} \vec{f}^{c}$ of contact forces resulting from the change to $\boldsymbol{F}+\mathrm{d} \boldsymbol{F}$ being determined, it remains to link the increment $\mathrm{d} \boldsymbol{F}$ to the increment $\mathrm{d} \boldsymbol{\sigma}$ of the Cauchy stress $\boldsymbol{\sigma}$, which according to the Cauchy-Poisson formula reads

$$
\boldsymbol{\sigma}=\frac{1}{|Y|} \sum_{c \in \mathcal{I}} \vec{f}^{c} \otimes \delta_{i}^{c} \vec{Y}^{i}
$$

where $|Y|$ denotes the surface of the base cell, $|Y|=\left\|\vec{Y}^{1} \times \vec{Y}^{2}\right\|$.

After a light amount of algebra, the differentiation of $\sigma$ reads

$$
\mathrm{d} \boldsymbol{\sigma}=\frac{1}{|Y|} \sum_{c \in \mathcal{I}} \delta_{i}^{c} \mathrm{~d} \vec{f}^{c} \otimes \vec{Y}^{i}+\boldsymbol{\sigma} \cdot \boldsymbol{F}^{-T} \cdot \mathrm{d} \boldsymbol{F}^{T}-\left(\boldsymbol{F}^{-T}: \mathrm{d} \boldsymbol{F}\right) \sigma,
$$

where $\boldsymbol{F}, \boldsymbol{\sigma}$, and $\vec{Y}^{i}$,s are known. The increments $\mathrm{d} \vec{f}^{c}$ are determined by solving the problem (22), which is linear; consequently, the increments $\mathrm{d} \vec{f}^{c}$ depend linearly on $\mathrm{d} \boldsymbol{F}$ and so does $\mathrm{d} \boldsymbol{\sigma}$. In that expression, $\mathrm{d} \boldsymbol{F}^{T}$ denotes the transposed of $\mathrm{d} \boldsymbol{F}$ and $\boldsymbol{F}^{-T}$ that of $\boldsymbol{F}^{-1}-\boldsymbol{F}^{-T}$ is also the inverse of $\boldsymbol{F}^{T}$.

\section{APPENDIX C : DETERMINATION OF THE “KRUYT” OPERATOR $C^{K A O}$}

The purpose of this appendix is to detail the generalization of the "Kruyt" operator $C^{U K O}$ of (13), in which the rotations of the grains and the preexisting contact forces are taken into account. Following the same idea, the displacements and 
the rotations of the grains "follow" the small homogeneous transformation of the macroscopic continuum medium. In particular, this means that, in general, the equilibrium equations are not satisfied so they do not have to be considered.

The displacement field of a homogeneously deformed continuous medium reads

$$
\vec{u}(\vec{x})=\vec{a}+\boldsymbol{E} \cdot \vec{x}
$$

therefore, obviously, $\boldsymbol{E}=\nabla_{x} \vec{u}$. To impose that the grains of the periodic medium "follow" the homogeneous transformation of the macroscopic continuum medium means that the displacements of the grain $n$ of the base cell is of the form $\vec{u}^{n}=\vec{a}+\boldsymbol{E} \cdot \vec{x}^{n}$ and that of the grain $n$ of the adjacent cell defined as $\left(v^{1}, v^{2}\right)$ is $\vec{u}^{\tilde{n}}=\vec{a}+\boldsymbol{E} \cdot\left(\vec{x}^{n}+v^{i} \vec{Y}_{a}^{i}\right)$, where $\boldsymbol{E}$ is the displacement gradient of the macroscopic continuous medium, that is to say, $\boldsymbol{E}=\mathrm{d} \boldsymbol{F} \cdot \boldsymbol{F}^{-1}$. Therefore, according to (B.3), the increment $\mathrm{d} \vec{b}^{c}$ of the branch vector $\vec{b}^{c}$ reads

$$
\mathrm{d} \vec{b}^{c}=\boldsymbol{E} \cdot \vec{b}^{c}=l^{c} \boldsymbol{E} \cdot \vec{e}^{c} .
$$

In the small strain assumption, the displacement field (C.1) of a continuous medium deforming homogeneously can be split into $\vec{u}(\vec{x})=\vec{a}+\boldsymbol{E}^{S} \cdot \vec{x}+\boldsymbol{E}^{A} \cdot \vec{x}$, where $\boldsymbol{E}^{S}=\frac{1}{2}\left(\boldsymbol{E}+\boldsymbol{E}^{T}\right)$ and $\boldsymbol{E}^{A}=\frac{1}{2}\left(\boldsymbol{E}-\boldsymbol{E}^{T}\right)$ are the symmetric and skew symmetric parts of $E \cdot \vec{x} \mapsto \vec{x}+\boldsymbol{E}^{A} \cdot \vec{x}$ is a small rotation. As the problem is $2 \mathrm{D}$, the skew symmetric tensor $\boldsymbol{E}^{A}$ is such that $\forall \vec{b}, \vec{b} \cdot \vec{i}_{3}=0, \boldsymbol{E}^{A} \cdot \vec{b}=\Omega \overrightarrow{i_{3}} \times \vec{b}$. Consequently, for any contact $c$, it comes $\boldsymbol{E}^{A} \cdot \vec{e}^{c}=\Omega \vec{i}_{3} \times \vec{e}^{c}=\Omega \vec{t}^{c}$ and $\Omega=\vec{t}^{c} .\left(\boldsymbol{E}^{A} \cdot \vec{e}^{c}\right)$.

To impose that the grain rotations of the base cell "follow" the homogeneous transformation of the macroscopic continuum medium comes down to impose that the rotations of the grains are all the same, namely, $\forall n, \theta^{n}=\Omega$.

The carrying of $\mathrm{d} \vec{b}^{c}=\boldsymbol{E} \cdot \vec{b}^{c}=l^{c} \boldsymbol{E} \cdot \vec{e}^{c}$ and $\theta^{n}=\Omega$ into the expression (B.11) of d $\vec{f}^{c}$ yields after some algebra

$$
\mathrm{d} \vec{f}^{c}=l^{c}\left(k_{n} \vec{e}^{c}\left(\vec{e}^{c} \otimes \vec{e}^{c}: \boldsymbol{E}\right)+\frac{k_{t}}{2} \vec{t}^{c}\left(\vec{t}^{c} \otimes \vec{e}^{c}+\vec{e}^{c} \otimes \vec{t}^{c}\right): \boldsymbol{E}\right)+\left(f_{n}^{c} \vec{t}^{c}-f_{t}^{c} \vec{e}^{c}\right)\left(\vec{t}^{c} \otimes \vec{e}^{c}: \boldsymbol{E}\right),
$$

or also taking into account the expression $\boldsymbol{E}=\mathrm{d} \boldsymbol{F} \cdot \boldsymbol{F}^{-1}$ as follows:

$$
\begin{aligned}
\mathrm{d} \vec{f}^{c}= & l^{c}\left(k_{n} \vec{e}^{c}\left(\vec{e}^{c} \otimes \vec{e}^{c} \cdot \boldsymbol{F}^{-T}\right): \mathrm{d} \boldsymbol{F}+\frac{k_{t}}{2} \vec{t}^{c}\left(\vec{t}^{c} \otimes \vec{e}^{c}+\vec{e}^{c} \otimes \vec{t}^{c}\right) \cdot \boldsymbol{F}^{-T}\right): \mathrm{d} \boldsymbol{F} \\
& +\left(f_{n}^{c} \vec{t}^{c}-f_{t}^{c} \vec{e}^{c}\right)\left(\vec{t}^{c} \otimes \vec{e}^{c} \cdot \boldsymbol{F}^{-T}\right): \mathrm{d} \boldsymbol{F} .
\end{aligned}
$$

\section{Direct determination of do}

Because the grains of the base cell are not in equilibrium in this analysis, it is not possible to use the expression (B.12) of $\mathrm{d} \boldsymbol{\sigma}$, and it is necessary to differentiate the more general expression as follows:

$$
\boldsymbol{\sigma}=\frac{1}{\left|Y_{a}\right|} \sum_{c \in \mathcal{I}} \vec{f}^{c} \otimes \vec{b}^{c}
$$

Taking into account the relations $\mathrm{d}\left|Y_{a}\right|=\left|Y_{a}\right| \boldsymbol{F}^{-T}: \mathrm{d} \boldsymbol{F}, \mathrm{d} \vec{b}^{c}=\mathrm{d} \boldsymbol{F} \cdot \boldsymbol{F}^{-1} \cdot \vec{b}^{c}$ and the expression (C.2) of $\mathrm{d} \vec{f}^{c}$, the differentiation of $\sigma$ yields after some algebra

$$
\begin{aligned}
\mathrm{d} \boldsymbol{\sigma}= & \frac{1}{\left|Y_{a}\right|} \sum_{c \in \mathcal{I}}\left(l^{c}\right)^{2} k_{n}\left(\left(\vec{e}^{c} \otimes \vec{e}^{c}\right) \otimes\left(\vec{e}^{c} \otimes \vec{e}^{c} \cdot \boldsymbol{F}^{-T}\right)\right) \cdot \mathrm{d} \boldsymbol{F} \\
& +\frac{1}{\left|Y_{a}\right|} \sum_{c \in \mathcal{I}}\left(l^{c}\right)^{2} \frac{k_{t}}{2}\left(\left(\vec{t}^{c} \otimes \vec{e}^{c}\right) \otimes\left(\left(\vec{t}^{c} \otimes \vec{e}^{c}+\vec{e}^{c} \otimes \vec{t}^{c}\right) \cdot \boldsymbol{F}^{-T}\right)\right) \cdot \mathrm{d} \boldsymbol{F} \\
& +\frac{1}{\left|Y_{a}\right|} \sum_{c \in \mathcal{I}} l^{c}\left(f_{n}^{c} \vec{t}^{c} \otimes \vec{e}^{c}-f_{t}^{c} \vec{e}^{c} \otimes \vec{e}^{c}\right) \otimes\left(\vec{t}^{c} \otimes \vec{e}^{c} \cdot \boldsymbol{F}^{-T}\right) \cdot \mathrm{d} \boldsymbol{F} \\
& -\left(\sigma \otimes \boldsymbol{F}^{-T}\right) \cdot \mathrm{d} \boldsymbol{F}+\sigma \cdot \boldsymbol{F}^{-T} \cdot \mathrm{d} \boldsymbol{F}^{T} .
\end{aligned}
$$

